\title{
Les substances responsables de l'arôme des fromages à pâte molle
}

\author{
par \\ J. ADDA et J.P. DUMONT \\ Laboratoire d'Etude des Arômes \\ C.N.R.Z - 78350 Jouy-en-Josas (France)
}

\section{INTRODUCTION}

Si la littérature abonde en travaux portant sur les substances responsables de l'arôme du Cheddar ou de l'Emmental, il n'en est pas de même pour les fromages à pâte molle. Constatant ce fait, dont il ne nous appartient pas d'apporter les raisons, nous nous sommes efforcés de rechercher et de réunir les travaux qui dans la littérature pourraient être utiles à une nécessaire mise au point. Dans ce cas particulier, notre ambition n'est pas tant de parvenir à une meilleure compréhension des mécanismes d'affinage que de susciter d'indispensables travaux complémentaires.

La notion de fromage à pâte molle est en elle-même assez imprécise, c'est pourquoi, afin de déterminer les limites de cette revue, nous avons choisi de nous référer au "catalogue préliminaire des différents types de fromages »(F.I.L., 1971). Cela nous a conduit à diviser notre étude en deux parties, relatives d'une part aux fromages à croûte lavée ou moisie, d'autre part aux fromages " bleus » fabriqués à partir de lait de vache (ce qui excluait certains fromages importants tels le Roquefort).

PREMIERE PARTIE

\section{LES FROMAGES A CROUTE LAVEe ET A CROUTE MOISIE}

\section{1) Acides gras libres}

Les acides aliphatiques et plus spécialement les termes volatils ont été largement étudiés dans les fromages à pâte molle. En effet, la lipolyse est particulièrement élevée dans ces fromages : c'est ainsi 
que dans le Camembert les acides gras libres peuvent atteindre 10 p. 100 des acides gras totaux (Kuzdzal-Savoie et Kuzdzal, 1966).

Les acides gras provenant de la lipolyse ont, comme les acides gras de la matière grasse de 4 à 20 atomes de carbone. Toutefois les proportions relatives des acides gras sont différentes dans les triglycérides et les acides gras libres car il s'opère une libération sélective des acides gras à longue chaîne (Hôte-Baudart, 1967). Ce phénomène a été maintes fois démontré en particulier pour le Camembert, le Pont-l'Evêque et le Coulommiers (Kuzdzal et Kuzdzal-Savoie, 1966) (tab. 1).

\section{TABLEAU 1}

Proportions relatives des acides gras dans les glycérides et les acides gras libres (en p. 100 du total)

\begin{tabular}{|c|c|c|c|c|c|c|}
\hline \multirow{2}{*}{$\begin{array}{l}\text { Désignation } \\
\text { des acides* }\end{array}$} & \multicolumn{2}{|c|}{ Pont-l'Evêque } & \multicolumn{2}{|c|}{ Camembert } & \multicolumn{2}{|c|}{ Coulommiers } \\
\hline & Glyc. & Ac. lib. & Glyc. & $\begin{array}{l}\text { Ac. lib. } \\
(10,6 \%)\end{array}$ & Glyc. & Ac. lib. \\
\hline $4: 0+6: 0$ & 3,5 & 3,9 & 2,8 & 2,2 & 2,5 & 4,0 \\
\hline $10: 0+$ gr $10: 0$ à $12: 0$ & 3,2 & 4,1 & 3,8 & 3,0 & 3,1 & 3,4 \\
\hline $12: 0+$ gr $12: 0$ à $14: 0$ & 4,3 & 4,4 & 4,5 & 4,7 & 4,2 & 4,6 \\
\hline $14: 0+$ gr $14: 0$ à $16: 0$ & 14,6 & 13,5 & 16,1 & 14,3 & 15,4 & 12,3 \\
\hline $16: 0+$ gr $16: 0$ à $18: 0$ & 27,6 & 24,8 & 28,6 & 23,3 & 27,5 & 23,7 \\
\hline $18: 0$ & 10,5 & 7,2 & 10,2 & 7,3 & 9,0 & 5,6 \\
\hline $18: 1+18: 2+18: 3$ & 36,3 & 42,1 & 34,0 & 45,2 & 38,3 & 46,4 \\
\hline
\end{tabular}

* Le premier chiffre indique le nombre d'atomes de carbone ; le second, le nombre de doubles liaisons.

L'abréviations gr signifie : groupe d'acides gras intermédiaires.

Kuzdzal-Savoie et Kuzdzal (1966).

L'intensité de la lipolyse varie suivant le type de fromage et également en fonction de sa maturité. Dans le cas du Camembert le degré d'acidité libre (exprimé en $\mathrm{ml}$ d'une solution IN $\mathrm{KOH}$ pour $100 \mathrm{~g}$ de matière grasse) qui est de 3,2 à 3,5 pour des fromages jeunes, passe à 6,1 pour des fromages affinés et atteint 13 pour les fromages surmaturés (Hôte-Baudart, 1967). Dans le Coulommiers la lipolyse semblerait moins intense (acidité libre de 2,3 pour un fromage aux trois quarts affiné). 
Pour le Munster (Kuzdzal-Savoie et al., 1971), l'étude des acides gras non-volatils montre que la lipolyse est toujours très élevée dans la croûte ( $5 \mathrm{~g}$ environ d'acides gras libres totaux pour $100 \mathrm{~g}$ d'extrait $\mathrm{sec}$ ), et variable dans la pâte, où la quantité d'acides non-volatils trouvée peut aller de $0,5 \mathrm{~g}$ à $6 \mathrm{~g}$ (pour $100 \mathrm{~g}$ d'extrait sec) suivant l'échantillon. Il ne semble pas dans ce cas que le degré de lipolyse constitue un facteur essentiel de la qualité organoleptique mais il est difficile de conclure de façon absolue compte tenu du très faible nombre d'échantillons examinés.

La lipolyse toutefois n'explique pas l'origine de tous les acides gras libres isolés, en particulier celle des termes volatils à chaîne ramifiée ou à chaîne droite très courte, dont la formation est attribuée au métabolisme microbien.

L'importance du phénomène est extrêmement variable d'un type de fromage à un autre. C'est ainsi que pour le Camembert 5 p. 100 seulement des acides gras libres totaux ne proviendraient pas de la lipolyse (Kuzdzal-Savoie et Kuzdzal, 1966).

Pour un certain nombre de types de fromages, l'identité des acides gras libres présents est bien connue. En dehors des acides butyrique et hexanoïque dont la présence peut être rattachée à la lipolyse on trouve à peu près toujours les acides acétique, propionique, isobutyrique et isovalérique (2-méthyl et 3-méthyl butyrique). Ils ont été mis en évidence dans le Camembert, le Munster, le Livarot, le Pont-l'Evêque et le Brie par Kuzdzal-Savoie et Kuzdzal (1971), dans le Camembert, le Livarot, le Pont-l'Evêque et le Brie par Stark et Adda (1972), le Carré de l'Est par Masson et Panouse (1968) et le Romadur par Schörmuller et Langner (1960).

L'acide valérique dont la présence est mise en doute par KuzdzalSavoie et Kuzdzal a pourtant été signalé dans le Romadur par Schörmuller et Langner (1960) et dans le Limbourg par Simonard et Mayaudon (1965).

L'acide isocaproïque identifié comme étant l'acide 3-méthyl valérique a été mis en évidence dans le Carré de l'Est par Masson et Panouse (1968), le Munster par Kuzdzal-Savoie et Kuzdzal (1971), le Livarot, le Pont-1'Evêque par Stark et Adda (1972) et le Romadur par Schörmuller et Langner (1960). Il semble être absent dans le Camembert (Stark et Adda, 1972 ; Kuzdzal-Savoie et Kuzdzal, 1971) et dans le Brie (Stark et Adda, 1972).

Les teneurs en chacun de ces acides sont très variables d'un type de fromage à un autre ; ceci apparaît dans le tableau 2 où figurent les poids d'acides gras volatils en $\mathrm{mg}$ pour $100 \mathrm{~g}$ de fromage humide. On constate qu'à l'intérieur d'un même type les écarts entre échantillons sont assez sensibles. Ces observations sont également valables pour les quantités globales d'acides gras volatils mises en jeu qui vont de $450 \mathrm{mg}$ pour $100 \mathrm{~g}$ de fromage frais dans le cas du Munster à $76 \mathrm{mg}$ dans le cas du Camembert. Les mesures de l'acidité volatile 
TABLEAU 2. - Dosage des acides gras libres volatils

\begin{tabular}{|c|c|c|c|c|c|c|c|c|c|c|c|}
\hline \multirow{2}{*}{$\begin{array}{c}\text { Type } \\
\text { de } \\
\text { fromage }\end{array}$} & \multirow{2}{*}{$\mathrm{N}^{\circ}$} & \multirow{2}{*}{ E.S. } & \multirow{2}{*}{ Gras/sec } & \multicolumn{7}{|c|}{ Acides gras libres volatils (en mg p. $100 \mathrm{~g}$ de fromage humide) } & \multirow{2}{*}{$\begin{array}{c}\text { Appréciation } \\
\text { du } \\
\text { goût }\end{array}$} \\
\hline & & & & $2: 0$ & $3: 0+i 4: 0$ & $4: 0$ & $\mathrm{i} 5: 0$ & $\mathrm{i} 6: 0$ & $6: 0$ & Total & \\
\hline Camembert & $\begin{array}{r}1 \\
2 \\
3 \\
4 \\
5 \\
6 \\
7 \\
8 \\
9 \\
10\end{array}$ & $\begin{array}{c}45,3 \\
48,2 \\
54,6 \\
- \\
- \\
- \\
- \\
45,0 \\
49,2 \\
46,1\end{array}$ & $\begin{array}{l}45 \\
45 \\
45 \\
50 \\
45 \\
50 \\
50 \\
45 \\
45\end{array}$ & $\begin{array}{l}2,0 \\
1,3 \\
6,6 \\
1,3 \\
2,0 \\
6,3 \\
3,8 \\
5,6 \\
5,5 \\
3,3\end{array}$ & $\begin{array}{r}2,0 \\
0,3 \\
13,7 \\
1,0 \\
0,3 \\
1,8 \\
3,3 \\
1,9 \\
1,4 \\
1,8\end{array}$ & $\begin{array}{r}12,0 \\
7,0 \\
20,6 \\
3,5 \\
4,3 \\
16,5 \\
7,3 \\
15,2 \\
18,6 \\
13,8\end{array}$ & $\begin{array}{r}0,8 \\
0,5 \\
21,4 \\
2,0 \\
0,8 \\
4,8 \\
2,0 \\
2,3 \\
1,8 \\
1,8\end{array}$ & $\begin{array}{l}- \\
- \\
- \\
- \\
- \\
- \\
\overline{0} \\
0,2\end{array}$ & $\begin{array}{r}7,8 \\
5,5 \\
14,3 \\
2,5 \\
2,3 \\
8,3 \\
3,8 \\
9,4 \\
10,7 \\
8,5\end{array}$ & $\begin{array}{r}24,6 \\
14,6 \\
76,6 \\
10,3 \\
9,7 \\
37,7 \\
20,2 \\
34,4 \\
38,0 \\
29,4\end{array}$ & $\begin{array}{c}++ \\
+ \\
+++ \\
- \\
- \\
- \\
- \\
++ \\
++ \\
++\end{array}$ \\
\hline Munster & $\begin{array}{l}11 \\
12 \\
12 \mathrm{a}\end{array}$ & $\frac{-}{-}$ & $\begin{array}{l}50 \\
50 \\
-\end{array}$ & $\begin{array}{l}124,8 \\
166,5 \\
192,1\end{array}$ & $\begin{array}{r}90,6 \\
120,3 \\
92,9\end{array}$ & $\begin{array}{r}107,5 \\
54,6 \\
47,4\end{array}$ & $\begin{array}{l}81,8 \\
49,7 \\
56,8^{*}\end{array}$ & $\begin{array}{l}47,5 \\
79,7 \\
45,5\end{array}$ & $\begin{array}{r}33,5 \\
6,3 \\
7,5\end{array}$ & $\begin{array}{l}485,7 \\
477,1 \\
443,3\end{array}$ & $\begin{array}{c}++ \\
+++ \\
++\end{array}$ \\
\hline Petit Munster & 13 & - & 45 & 139,0 & 266,6 & 236,0 & 198,0 & - & 18,0 & 857,6 & ++ \\
\hline Livarot & 14 & - & - & 74,3 & 136,4 & 32,7 & 45,5 & 27,8 & 10,3 & 327,0 & ++ \\
\hline Petit Livarot & 15 & 52,1 & - & 26,7 & 39,2 & 29,3 & 46,7 & 7,8 & 1,4 & 151,1 & +++ \\
\hline Pont-l'Evêque & 19 & - & - & 16,5 & 28,0 & 5,3 & 17,0 & 3,5 & 3,0 & 73,3 & ++ \\
\hline Brie & 22 & - & - & 7,5 & 5,3 & 10,5 & 5,0 & 0,3 & 3,5 & 32,1 & ++ \\
\hline Vacherin (Suisse) & 25 & - & - & 7,0 & 11,0 & 4,8 & 2,4 & 0,4 & 2,8 & 28,4 & +++ \\
\hline
\end{tabular}

- Goût défectueux ; + Léger défaut de goût ; ++ Assez bon goût ; +++ Très bon goût.

2:0 = acide acétique ; $3: 0=$ acide propionique ; i4:0 = acide isobutyrique ; 4:0 = acide butyrique ; $15: 0=$ acide isovalérique ; $5: 0=$ acide valérique $; 16: 0=$ acide isocaproïque $; 6: 0=$ acide caproïque.

Tiré de Kuzdzal-Savoie et Kuzdzal (1971). 
(Simonard et Mayaudon, 1956) traduisent les mêmes tendances (l'acidité volatile exprimée en mE pour $1000 \mathrm{~g}$ atteint 13,5 pour un Limbourg de 6 semaines alors qu'elle ne dépasse pas 1,3 pour le Camembert).

Parallèlement, la qualité du Camembert ne semble pas dépendre uniquement de la quantité d'acides volatils présente puisque pour deux échantillons de très bonne qualité organoleptique (tab. 2, échantillons 3 et 8 ) la quantité totale d'acides volatils peut varier du simple au double tandis que les appréciations organoleptiques portées sur deux échantillons contenant les différents acides en quantités pratiquement égales sont très sensiblement différentes (échantillons 6 et 8 ). Les valeurs figurant dans ce tableau s'écartent notablement de celles citées par Schörmuller et Langner (1960) pour le Camembert. Ces auteurs mentionnent (tab. 3 ) des taux nettement plus élevés pour l'acide acétique $(30 \mathrm{mg} / 100 \mathrm{~g})$, l'acide isobutyrique $(53 \mathrm{mg} / 100 \mathrm{~g})$ et l'acide hexanoïque ( $29 \mathrm{mg} / 100 \mathrm{~g}$ ) et ne signalent pas la présence d'acide isovalérique alors qu'ils mentionnent son existence dans le Romadur.

La présence d'acides aromatiques a été signalée dans plusieurs types de fromages : les acides benzoïque et phénylacétique ont été isolés et identifiés dans le Camembert et le Limbourg (Simonart et Mayaudon, 1956), le Livarot et le Munster (Kuzdzal-Savoie, 1971).

L'acide hydroxybenzoïque est présent dans le Camembert (Simonart et Mayaudon, 1956) et le Romadur ; dans ce dernier Kiermeir

TABLEAU 3

\begin{tabular}{c|c|c}
\hline & Romadur & Camembert \\
\hline $\mathrm{C}_{2}$ & 196,80 & 30,00 \\
$\mathrm{C}_{3}$ & 94,72 & 2,96 \\
$\mathrm{C}_{4}$ & 13,92 & 26,40 \\
$\mathrm{iC}_{4}$ & 63,36 & 53,68 \\
$\alpha \mathrm{Me} \mathrm{But}_{\mathrm{C}}$ & 16,32 & \\
$\mathrm{C}_{5}$ & 24,48 & 0 \\
$\mathrm{iC}_{5}$ & 13,06 & 29,00 \\
$\mathrm{C}_{8}$ & 13,92 & \\
$\mathrm{iC}_{6}$ & 46,40 & \\
\hline
\end{tabular}


et Jarczynski (1960) ont trouvé des doses allant de 0,1 mg pour $100 \mathrm{~g}$ pour un fromage âgé de $9 \mathrm{j}$ à $0,4 \mathrm{mg}$ pour $100 \mathrm{~g}$ pour un fromage âgé de $30 \mathrm{j}$.

L'existence d'acide hydroxyphénylacétique a été signalée dans le Camembert et le Limbourg par Simonart et Mayaudon (1956), dans le Camembert par Schörmuller et Langner (1960) tandis que celle de l'acide phényl propionique l'a été dans le Munster, le Livarot (Kuzdzal et al., 1971) et le Limbourg. L'acide hydroxyphénylpropionique a été identifié dans le Camembert par Schörmuller et Langner (1960) et le Limbourg (Simonart et Mayaudon, 1956).

\section{2) Composés carbonyles}

Les travaux portant sur l'étude des composés carbonylés dans les fromages à croûte moisie ou lavée sont très peu nombreux. Les seules données quantitatives sont celles publiées par Schwartz et Parks (1963) qui comparent trois Camemberts d'origines différentes. On peut voir (tab. 4) que l'acétone n'apparaît jamais et que la 2-pentanone

\section{TABLEAU 4}

Concentration en méthylcétones dans la matière grasse extraite de Camembert d'origines diverses ( $\mu$ mole pour $10 \mathrm{~g}$ de m.g.)

\begin{tabular}{|c|c|c|c|c|c|c|}
\hline Pays d'origine & $\left(C_{15}+C_{13}\right)$ & $\mathrm{C}_{11}$ & $\mathrm{C}_{\mathrm{r}}$ & $\mathrm{C}_{7}$ & $\mathrm{C}_{5}$ & Total \\
\hline France & $<0,1$ & 0,68 & 3,37 & 1,53 & 0,34 & 5,92 \\
\hline Danemark & $<0,1$ & 0,54 & 1,38 & 1,05 & 0,35 & 3,32 \\
\hline U.S.A. & $<0,1$ & 0,48 & 1,45 & 0,60 & 0,00 & 2,53 \\
\hline
\end{tabular}

Tiré de Schwartz et Parks (1963).

est absente dans l'un des échantillons. Observation à rapprocher de celles de Dolezalek et Brabcora (1964) qui rapportent que dans les fromages à croûte moisie du type Brie et Camembert toutes les aldéhydes et cétones sont présentes dès le $8^{\mathrm{e}}$ jour d'affinage mais que des fromages trop affinés, qui ne possèdent d'ailleurs plus un arôme typique, ne contiennent pas les méthylcétones en $\mathrm{C}_{5}, \mathrm{C}_{\tau}$ et $\mathrm{C}$.

A l'occasion d'un travail analogue au précédent mais portant sur le Limbourg, Tuckey et al. (1959) notent que tous les composés carbonyles présents au premier jour d'affinage le sont en fin d'affinage et comme la quantité de ces composés n'augmente pas, il semble qu'en définitive l'arôme du Limbourg soit indépendant des composés 
carbonyles. Cette dernière observation est également formulée par Grill et al. (1966) pour le Trappiste.

Sur le plan qualitatif la 2-nonanone a été identifiée dans le Livarot (Ramshaw et Adda) et dans le Pont-l'Evêque (Dumont et Adda), fromage qui semble contenir également la 2-heptanone et la 2-pentadécanone. Par ailleurs, la 2-pentanone, la 2-hexanone, la 2-heptanone, la 2-undecanone, la 2-tridecanone et la 2-pentadecanone ont également été identifiées dans une pâte molle stabilisée (Dumont et Adda).

Ces mêmes auteurs mettent en évidence l'acétone, la 2-pentanone et la 2-heptanone dans le Munster.

Le diacétyle semble, selon Csiszar et al. (1956) présent en faible quantité $(0,05 \mathrm{ml}$ pour $100 \mathrm{~g}$ de fromage) dans le Camembert ainsi que dans les pâtes molles stabilisées (Dumont et Adda). L'acétophénone a été mise en évidence dans le Livarot (Ramshaw et Adda) et le Pont-l'Evêque (Dumont et Adda).

\section{3) Alcools}

L'éthanol, le 2-propanol, le 2-butanol, le 2-octanol et le 2-nonanol ont été mis en évidence dans les pâtes molles stabilisées (Dumont et Adda), l'alcool isoamylique semble être présent dans le Livarot (Ramshaw et Adda) et le Munster (Dumont et Adda) ; le 2-pentanol a été trouvé dans le Munster et les pâtes stabilisées tandis que le 2-heptanol n'a été trouvé que dans le Munster.

L'Acétoïne semble être présente dans le Camembert et le Romadur (Csiszar et al., 1956) à des doses de l'ordre de $3 \mathrm{mg} / 100 \mathrm{~g}$ de fromage. Le 2-phényl éthanol a été mis en évidence dans le Livarot (Ramshaw et Adda) mais semblerait également être présent dans le Camembert et l'Epoisses (Dumont et Adda).

\section{4) Phénols}

Le phénol a été mis en évidence dans le Livarot et le Pontl'Evêque tandis que les $\mathrm{m}$ et $\mathrm{p}$ crésols l'ont été dans le Livarot seul (Ramshaw et Adda). Ces composés semblent jouer un rôle important dans l'arôme de ces fromages.

\section{5) Composés soufrés}

Les composés soufrés semblent particulièrement importants au point de vue organoleptique dans les fromages à croûte lavée. L'hydrogène sulfuré et le méthylmercaptan ont été mis en évidence et dosés dans le Trappiste, où ils seraient présents à raison de 1,9 et 2,3 ppm respectivement (Grill et al., 1966).

Le disulfure de diméthyle est mis en évidence dans les fromages à croûte lavée comme le Livarot (Ramshaw et Adda) et le Munster (Dumont et Adda). 


\section{6) Hydrocarbures}

Un certain nombre d'hydrocarbures est régulièrement mis en évidence quel que soit le type de fromage examiné. Ainsi le toluène dans le Livarot et le Pont-l'Evêque (Ramshaw et Adda), dans le Pontl'Evêque et le Munster (Dumont et Adda) ; le diméthyl naphtalène sur les pâtes molles stabilisées (Dumont et Adda). Des hydrocarbures aliphatiques ont également été rencontrés à plusieurs reprises par ces derniers dans divers types de fromages.

\section{7) Esters}

Les esters semblent être présents dans les différents types de fromages bien qu'aucune publication n'en fasse mention. Des esters aromatiques ont été trouvés dans le Livarot (Ramshaw et Adda), tandis que des esters éthyliques d'acides gras à 10 et 12 atomes de carbone ont été identifiés dans divers échantillons (Dumont et Adda).

\section{8) Lactones}

Les deca et dodecalactones ont été identifiées dans le Pont-l'Evêque et dans une pâte molle stabilisée (Dumont et Adda).

\section{9) Acides aminés}

Des essais portant sur 10 échantillons de fromage affiné de type Camembert fabriqué à partir de lait cru (Lenoir, 1963), montrent que l'azote ammoniacal constitue de 21 à 27 p. 100 de l'azote soluble (7,73 p. 100 de l'azote total), résultats qui concordent avec les valeurs publiées par Tsugo (1959) qui estime que 25 p. 100 environ de l'azote soluble est sous forme ammoniacale $(2,4$ à $3,7 \mathrm{mg} \mathrm{N}$-ammoniacal/g).

Selon Jacquet et Lenoir (1954) l'ammoniac apparaîtrait dans le Camembert vers le $18^{\mathrm{e}}$ jour de l'affinage et sa teneur augmenterait rapidement ensuite.

En ce qui concerne l'azote aminé les valeurs de 9 à 12 p. 100 avancées par Lenoir (1963) concordent avec celles publiées par Hänni (1967) (l'azote aminé représenterait 6 à 12 p. 100 de l'azote total dans le Camembert et le Brie, 5 à 10 p. 100 dans le Munster et 5 à 13 p. 100 dans le Limbourg) exprimées en poids ; ceci représente dans le cas du Camembert, de 0,84 à 1,59 $\mathrm{g}$ d'acides aminés libres pour $100 \mathrm{~g}$ de fromage. Hänni (1967), quant à lui, estime la teneur en acides aminés libres du Brie, du Limbourg et du Camembert à 1,4 $\mathrm{g}$, $0,9 \mathrm{~g}$ et $0,6 \mathrm{~g} / 100 \mathrm{~g}$ respectivement, valeurs bien moins élevées que celles obtenues par le calcul à partir des résultats présentés par Kosikowski et Dahlberg (1954) pour le Camembert. Dans leur travail 
sur le Limbourg, Tuckey et Sahasrabudhe (1957) donnent des valeurs voisines de 1,4 g/100 g à l'âge de 10 semaines et notent l'absence de corrélation entre la présence d'un acide aminé donné et l'arôme du fromage. Ces valeurs sont du même ordre de grandeur que celles citées par Kosikowski et Dahlberg pour le Limbourg, le Liederkranz et le Brick.

\section{0) Amines}

\section{a) Amines volatiles}

Ney et Wirotama (1971) étudiant des Camemberts fabriqués soit en France, soit en Allemagne mettent en évidence de nombreuses amines : Méthylamine, Ethylamine, n-propylamine, isopropylamine, n-butylamine, l-méthylpropylamine, n-amylamine, iso-amylamine, anteiso-amylamine, n-hexylamine, éthanolamine, diméthylamine, diéthylamine, dipropylamine et dibutylamine, mais ne donnent aucune indication sur les quantités présentes.

\section{b) Amines non-volatiles}

La Tyramine a été dosée dans un certain nombre de types de fromages. Les résultats publiés sont regroupés dans le tableau 5 . De plus Bulinski (1969) a publié les dosages effectués sur le Camembert pour l'histamine $(209 \mu \mathrm{g} / \mathrm{g})$, la tryptamine $(20 \mu \mathrm{g} / \mathrm{g})$ et la putrescine $(92 \mu \mathrm{g} / \mathrm{g})$.

\section{TABLEAU 5}

Teneur de quelques types de fromages en Tyramine $(\mu \mathrm{g} / \mathrm{g})$

\begin{tabular}{r|c|c|c|c}
\hline Camembert & Brie & Limbourg & Brick & Boursault \\
\hline $86(1)$ & $180(1)$ & $204(2)$ & $524(3)$ & $1116(3)$ \\
$125(2)$ & & $120(4)$ & & \\
$23(3)$ & & & \\
$2000(4)$ & & & \\
$90-124(4)$ & & & \\
\hline
\end{tabular}

(1) Horowitz et al. (1964).

(2) Dahlberg et Kosikowski (1948).

(3) Sen (1969).

(4) Bulinski (1969). 
DEUXIEME PARTIE

\section{LES FROMAGES A PATE PERSILLEE}

\section{1) Les acides gras libres}

Les acides gras libres jouent un rôle important dans l'arôme des pâtes persillées. C'est ainsi que Niki et al. (1966) ont noté que les souches les mieux adaptées à la production de fromages Bleus de qualité sont celles qui présentent une forte activité lipolytique accompagnée d'une faible activité protéolytique.

TABLEAU 6

Acides gras libres dans le fromage Bleu* (mg d'acide $/ \mathrm{kg}$ de fromage)

\begin{tabular}{c|r|c|c}
\hline & \multicolumn{3}{|c}{ Echantillons } \\
\cline { 2 - 3 } Acide & \multicolumn{1}{|c}{ A } & B & C \\
\hline $2: 0$ & & & \\
$4: 0$ & 1417 & 715 & 345 \\
$6: 0$ & 1269 & 558 & 2517 \\
$8: 0$ & 887 & 369 & 1471 \\
$10: 0$ & 786 & 291 & 1237 \\
$12: 0$ & 1414 & 514 & 2026 \\
$14: 0$ & 1686 & 619 & 2458 \\
$16: 0$ & 6199 & 2044 & 9324 \\
$18: 0$ & 13212 & 4848 & 20308 \\
$18: 1$ & 4880 & 1610 & 6239 \\
$18: 2$ & 14347 & 4819 & 18199 \\
$18: 3$ & 1336 & 266 & 1615 \\
& 1358 & 283 & 1320 \\
\hline
\end{tabular}

* Moyenne de deux analyses. 
L'indice d'acidité, exprimé en $\mathrm{mg} \mathrm{KOH} / \mathrm{g}$ de matière grasse a été mesuré par Svensen et Ottestad (1969) sur 22 échantillons de Normanna. Les résultats varient de 10,6 à 37,8 mais semblent indépendants de l'âge des échantillons. Pour un même fromage l'indice augmente de 14 unités entre la surface et le centre du fromage.

Comme dans le cas des fromages de type Camembert, la lipolyse libère surtout des acides gras à longue chaîne (travaux de Benassi (1964) sur le Gorgonzola et d'Anderson et Day (1965) pour le Bleu). Des variations importantes sont enregistrées d'un fromage à l'autre (tab. 6). On peut remarquer qu'il ne figure pas d'acides gras ramifiés ni d'acides formique et propionique parmi les acides gras volatils. Signalons cependant que d'autres auteurs signalent la présence de ces acides ramifiés. Ainsi Schörmuller et Langner (1960) relatent la présence d'acide isobutyrique dans le Gorgonzola (tab. 7). De leur côté Coffman et al. (1960) signalent la présence d'acide isovalérique dans le fromage Bleu en poudre, tandis que Ney et Wiramata (1972) signalent la présence d'acide isobutyrique dans l'Edelpilzkäse aux côtés des acides acétique, propionique, butyrique et valérique.

TABLEAU 7

Acides gras volatils dans quelques types de Bleus (Résultats exprimés en $\mathrm{mg} / 100 \mathrm{~g}$ de fromage frais)

\begin{tabular}{|c|c|c|c|c|}
\hline & Gorgonzola* & $\begin{array}{c}\text { Bleu** } \\
\text { d'Auvergne }\end{array}$ & $\begin{array}{l}\text { Bleu** } \\
\text { Europe }\end{array}$ & Bleu**** \\
\hline $\mathrm{C}_{2}$ & 288 & 1,3 & 11,3 & 82,6 \\
\hline $\mathrm{C}_{3}$ & 23,68 & 3,3 & 5,5 & - \\
\hline $\mathrm{iC}_{4}$ & 130,24 & & & \\
\hline $\mathrm{C}_{4}$ & 38,72 & 5,3 & 61,0 & 144,8 \\
\hline $\mathrm{iC}_{5}$ & & 10,0 & 12,5 & \\
\hline $\mathrm{C}_{5}$ & 24,48 & & & \\
\hline $\mathrm{iC}_{6}$ & 23,20 & - & 0,1 & \\
\hline $\mathrm{C}_{6}$ & 51,94 & 3,0 & 21,5 & 90,9 \\
\hline $\begin{array}{l}* \text { Schi } \\
* * \text { Kuz } \\
* \text { And }\end{array}$ & $\begin{array}{l}\text { et Langner } \\
\text { oie et Kuzdza } \\
966) \text {. }\end{array}$ & $\begin{array}{l}\text { 971). } \\
\text {. }\end{array}$ & & \\
\hline
\end{tabular}


Kuzdzal-Savoie et Kuzdzal (1971) notent la présence dans le Bleu d'Auvergne d'acide isovalérique et semble-t-il d'acide isobutyrique (ne séparant pas acide propionique et acide isobutyrique dans leurs résultats il semble difficile de conclure) (tab. 7.).

Dans le Stilton, Berridge et al. (1953) constatent l'apparition précoce et importante d'acide acétique qui, bien que tendant à diminuer ultérieurement à mesure que la moisissure se développe n'en demeure par moins présent en quantité appréciable dans le fromage affiné où il voisine avec les acides butyrique, caproïque, caprylique et caprique.

\section{2) Les composés carbonylés}

\subsection{Cétones}

De nombreuses cétones ont été mises en évidence dans les pâtes persillées. Patton (1950) note la présence d'acétone, de 2-pentanone, 2-heptanone et 2-nonanone, liste à laquelle Morgan et Anderson (1956) étudiant le Roquefort, le Bleu danois et le Gorgonzola ajoutent la 2-butanone.

Anderson (1966) utilisant la spectrométrie de masse identifie avec certitude la série homologue des méthylcétones de 5 à 13 atomes de carbone et pense avoir mis en évidence la présence de diacétyle et d'acétone.

Svensen et Ottestad (1969) mettent en évidence dans le Normanna la série homologue des méthylcétones à nombre impair d'atomes de carbone de $\mathrm{C}_{3}$ à $\mathrm{C}_{13}$ ainsi que la 2-nonenone qu'ils sont seuls à avoir signalée. Sur un lot de 22 échantillons âgés de 50 à $380 \mathrm{j}$, ils observent des variations individuelles considérables qui semblent indépendantes de l'âge des échantillons. Les valeurs données (tab. 8) sont du même ordre de grandeur que celles publiées antérieurement par Anderson et Day (1966) pour 5 échantillons de Bleu (tab. 9).

\section{TABLEAU 8}

Teneurs moyennes en cétones de 22 échantillons de fromage Normanna* (Exprimées en $\mathrm{mg} / 200 \mathrm{~g}$ de fromage)

\begin{tabular}{|c|c|c|c|c|c|}
\hline & $\mathrm{C}_{5}$ & $\mathrm{C}_{7}$ & $\mathrm{C}_{9}$ & $C_{9,11}$ & $\mathrm{C}_{11}$ \\
\hline Moyenne & 3,50 & 7,50 & 12,80 & 1,94 & 0,68 \\
\hline Valeurs extrêmes & $0,26-28,44$ & $0,96-19,39$ & $0,79-28,94$ & $<0,1-4,06$ & $<0,1-4,06$ \\
\hline
\end{tabular}

* Svensen et Ottestad (1969). 


\section{TABLEAU 9}

Teneurs de 5 échantillons de fromage Bleu en méthylcétones* (Exprimées en $\mathrm{mg} / \mathrm{kg}$ de fromage)

\begin{tabular}{|c|c|c|c|c|c|}
\hline Echantillon & $\mathrm{C}_{3}$ & $\mathrm{C}_{5}$ & $\mathrm{C}_{\overline{7}}$ & $\mathrm{C}_{b}$ & $\mathrm{C}_{11}$ \\
\hline A & 3,4 & 18,4 & 40,8 & 28,0 & 6,4 \\
\hline B & 2,8 & 7,2 & 19,0 & 22,3 & 6,0 \\
\hline $\mathrm{C}$ & 3,9 & 20,9 & 71,8 & 88,3 & 29,9 \\
\hline D & 1,7 & 6,5 & 17,9 & 19,8 & 4,9 \\
\hline $\mathrm{E}$ & 2,7 & 17,5 & 39,1 & 42,5 & 12,9 \\
\hline
\end{tabular}

* Anderson et Day (1966).

Etudiant l'évolution du taux des méthylcétones au cours de l'affinage, Dartey et Kinsella (1971) notent qu'il croît régulièrement jusque vers le $70^{\mathrm{e}}$ jour (il passe de 1,86 à 120,4 micromoles pour $10 \mathrm{~g}$ d'extrait sec entre le $10^{\circ}$ et le $70^{\mathrm{e}}$ jour), puis constatent une décroissance entre le $70^{\mathrm{e}}$ et le $100^{\mathrm{e}}$ jour. Les concentrations relatives de chacune des méthylcétones semblent fluctuer assez largement mais il apparaît que celles de la 2-heptanone et de la 2-nonanone augmentent régulièrement.

Une cinétique d'affinage quelque peu identique a été observée dans certains cas par Sato et al. (1966) : au cours d'une expérience permettant la comparaison de 5 souches de Penicillium roqueforti ils constatent que, de façon générale, la quantité de carbonyles augmente avec l'âge des fromages, mais qu'elle dépend largement de la souche utilisée et surtout du bon développement de celle-ci. Dans certains cas les teneurs en 2-heptanone et 2-nonanone atteignent un maximum $(9 \mu \mathrm{g} / \mathrm{g})$ après 1 mois d'affinage puis décroissent rapidement. Dans d'autres cas, au contraire, il y a augmentation régulière au cours des 3 mois d'affinage, les valeurs maximum atteintes étant alors fonction du bon développement de la souche : les méthylcétones peuvent être à peine décelables dans les cas extrêmes, alors que la 2-nonanone peut être présente à raison de $10 \mu \mathrm{g} / \mathrm{g}$ dans d'autres cas.

Les résultats de Schwartz et Parks (1963) montrent (tab. 10) une large variabilité entre échantillons, indépendante semble-t-il de l'âge de ces derniers. Ces auteurs soulignent que la 2-heptanone est toujours le composé quantitativement le plus important, assertion qui vient en contradiction avec certains résultats présentés précédemment, mais qui est en accord avec ceux présentés par Anderson (1966). 


\section{TABLEAU 10}

Méthylcétones dans la matière grasse de fromage Bleu (Résultats exprimés en $\mu \mathrm{m}$ pour $10 \mathrm{~g}$ de M.G.)

\begin{tabular}{|c|c|c|c|c|c|c|c|}
\hline Echantillon & Age de l'échantillon & $\left(C_{15}+C_{13}\right)$ & $\mathrm{C}_{11}$ & $\mathrm{C}_{3}$ & $\mathrm{C}_{7}$ & $\mathrm{C}_{5}$ & $\mathrm{C}_{3}$ \\
\hline$A^{*}$ & 2 mois minimum & 1,1 & 1,7 & 7,6 & 30,2 & 20,0 & 5,1 \\
\hline $\mathrm{B}^{*}$ & 3 mois minimum & 0,8 & 0,7 & 2,5 & 3,4 & 0,9 & trace \\
\hline$C^{*}$ & 4 mois minimum & 1,3 & 1,7 & 8,5 & 12,4 & 7,2 & trace \\
\hline $\mathrm{D}^{* *}$ & - & & 2,4 & 9,3 & 11,2 & 5,9 & 1,7 \\
\hline
\end{tabular}

* Schwartz et Parks (1963).

** Anderson (1966). Moyenne de 3 échantillons.

\subsection{Aldéhydes}

Anderson (1966) identifie l'éthanal, la 3-méthylbutanal, l'isobutanal et le furfural dans le Bleu tandis que Ney et Wirotama (1972) étudiant l'Edelpilzkäse mettent en évidence éthanal, propional, butanal, isobutanal, pentanal, isopentanal et la phénylacétaldéhyde.

\section{3) Alcools}

Jackson et Hussong (1958) mettent en évidence la formation d'alcools secondaires (2-pentanol, 2-heptanol, et 2-nonanol) mais ceux-ci n'apparaissent que lorsque le fromage contient une forte quantité de méthylcétones. Ces alcools secondaires ont été dosés par Anderson et Day (1966) sur quelques échantillons. Les résultats présentés (tab. 11) sont en accord avec les valeurs données par Svensen et Ottestad (1969) sur un lot de 22 échantillons de Normanna (tab. 12). On peut remarquer que ces derniers auteurs mettent également en évidence l'éthanol, les 2-méthyl et 3-méthylbutanol, le 2-nonenol et le 2-undecanol.

Dans une étude plus fine, Anderson (1966) identifie avec certitude par spectrométrie de masse une série d'alcools secondaires (propanol, pentanol, heptanol, octanol, nonanol), le 2-méthylbutanol, le 3-méthylbutanol et le 1-pentanol, et pense avoir identifié le méthanol, l'éthanol et le 2-phényléthanol, ce dernier composé semblant être très important au point de vue organoleptique. 


\section{TABLEAU 11}

Concentration de fromage de type Bleu en alcools secondaires

(Résultats exprimés en $\mathrm{mg} / \mathrm{kg}$ de fromage)*

\begin{tabular}{|c|c|c|c|c|c|}
\hline & A & B & C & D & $\mathrm{E}$ \\
\hline$C_{5}$ & 0,6 & 0,3 & 1,9 & 1,3 & 0,3 \\
\hline $\mathrm{C}_{7}$ & 6,3 & 8,2 & 9,1 & 9,8 & 3,0 \\
\hline $\mathrm{C}_{2}$ & 3,9 & 3,7 & 4,3 & 2,7 & 2,5 \\
\hline
\end{tabular}

* Moyenne de deux analyses.

Anderson et Day (1966).

TABLEAU 12

Concentration en alcools du fromage Normanna

(Résultats exprimés en mg p. $200 \mathrm{~g}$ de fromage)

\begin{tabular}{|c|c|c|c|c|}
\hline & $\mathrm{C}_{5}$ & iso $\mathrm{C}_{5}$ & $\mathrm{C}_{7}$ & $\mathrm{C}_{\ominus}$ \\
\hline Moyenne* & 0,54 & 0,45 & 1,39 & 0,67 \\
\hline Valeurs extrêmes & $0,1-1,72$ & $0,1-0,96$ & $0,26-3,04$ & $0,1-2,46$ \\
\hline
\end{tabular}

Calculée sur 22 échantillons.

Svensen et Ottestad (1969).

\section{4) Esters}

Un certain nombre d'esters d'acides aliphatiques ont été identifiés dans le Bleu par Anderson (1966) ce sont les esters méthyliques des acides butanoïque, hexanoïque, octanoïque, décanoïque et dodécanoïque, les esters éthyliques des acides formique, acétique, butanoïque, hexanoïque, octanoïque et décanoïque. De plus, ont été identifiés avec moins de certitude l'acétate de méthyle, l'hexanoate d'isopropyle, le butanoate de 3-méthylpropyle, l'hexanoate de pentyle, le nonanoate de 2-méthyléthyle (isopropyle), et le décanoate d'isopropyle. 
Ney et Wirotama (1972) signalent la présence des esters éthyliques des acides butanoïque, hexanoïque, octanoïque et décanoïque dans l'Edelpilzkäse.

\section{5) Autres composés neutres}

Les lactones en $\mathrm{C}_{8}$ et $\mathrm{C}_{10}$ ont été identifiées par Anderson qui a, en outre, mis en évidence le méthylmercaptan et l'hydrogène sulfuré parmi les composés soufrés et le benzène et le toluène parmi les hydrocarbures.

\section{6) Acides aminés}

Les acides aminés ont été étudiés par Kosikowski et Dahlberg (1954) dans le Gorgonzola et le Bleu. Les résultats apparaissent dans le tableau 13 et montrent que les taux d'acide glutamique et de valine sont particulièrement élevés atteignant ou dépassant $6 \mathrm{mg}$ par gramme de fromage.

\section{TABLEAU 13}

Acides aminés libres dans les fromages Bleus $(\mathrm{mg} / \mathrm{g})$

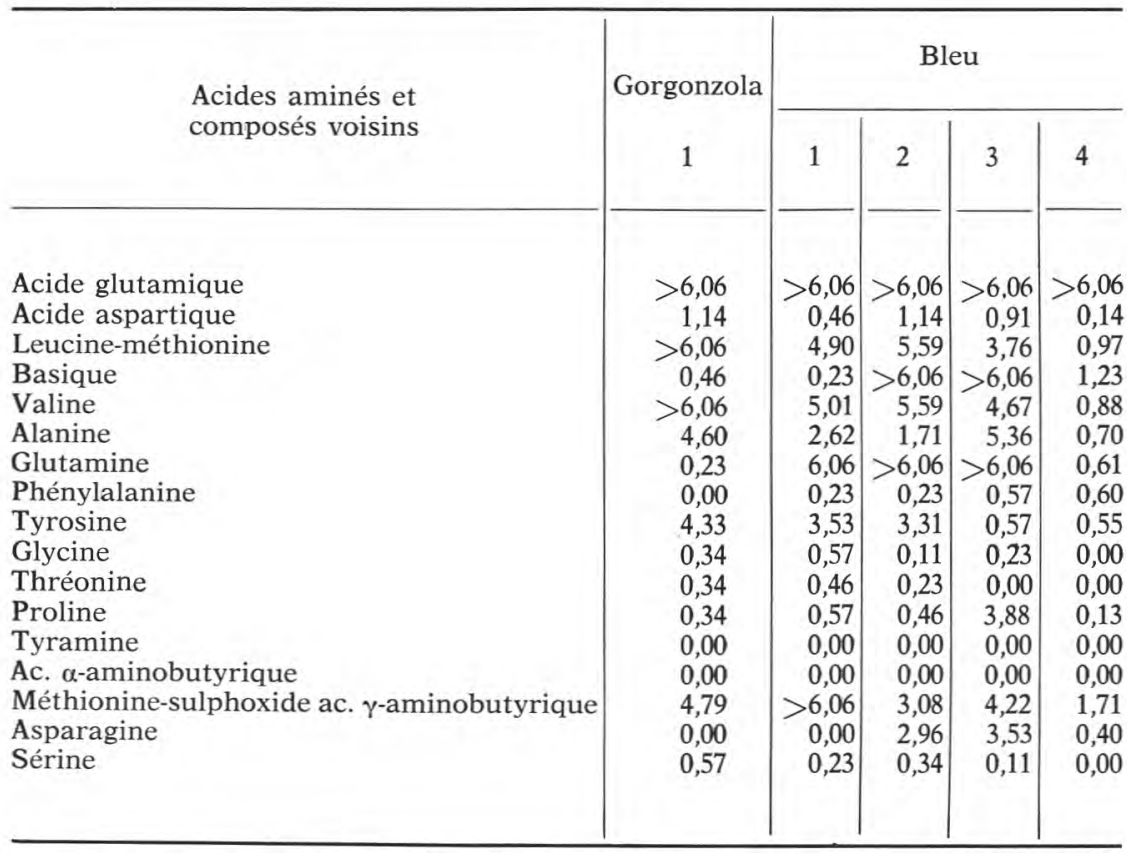

D'après Kosikowski et Dahlberg (1954). 


\section{7) Amines}

a) Amines volatiles

Spettoli (1971) indique les taux (exprimés en $\mu \mathrm{g} / \mathrm{g}$ ) de quelques amines volatiles pour 2 échantillons de Gorgonzola.

\begin{tabular}{c|c|c|c|c|c}
\hline & $\begin{array}{c}\text { Amines vol. } \\
\text { totales }\end{array}$ & Hexylamine & Isoamylamine & Isobutylamine & Ethylamine \\
\hline I & 6,4 & 0,7 & 1,5 & 2,5 & 1,7 \\
II & 132,4 & 0 & 12,4 & 0 & 120 \\
\hline
\end{tabular}

Ney et Wiromata (1972) travaillant sur l'Edelpilzkäse mettent en évidence les méthylamine, éthylamine, n-butylamine, isobutylamine, isopentylamine, diméthylamine, diéthylamine di-n-propylamine et di-n-butylamine mais ne donnent aucun résultat quantitatif.

b) Amines non-volatiles

Les renseignements fournis par la littérature sont plus nombreux que dans les amines volatiles et intéressent principalement la tyramine. Ces résultats, qui témoignent d'une très grande variabilité, sont résumés dans le tableau 14.

\section{TABLEAU 14}

Teneurs en Tyramine de différents fromages (Résultats exprimés en $\mu \mathrm{g} / \mathrm{g}$ )

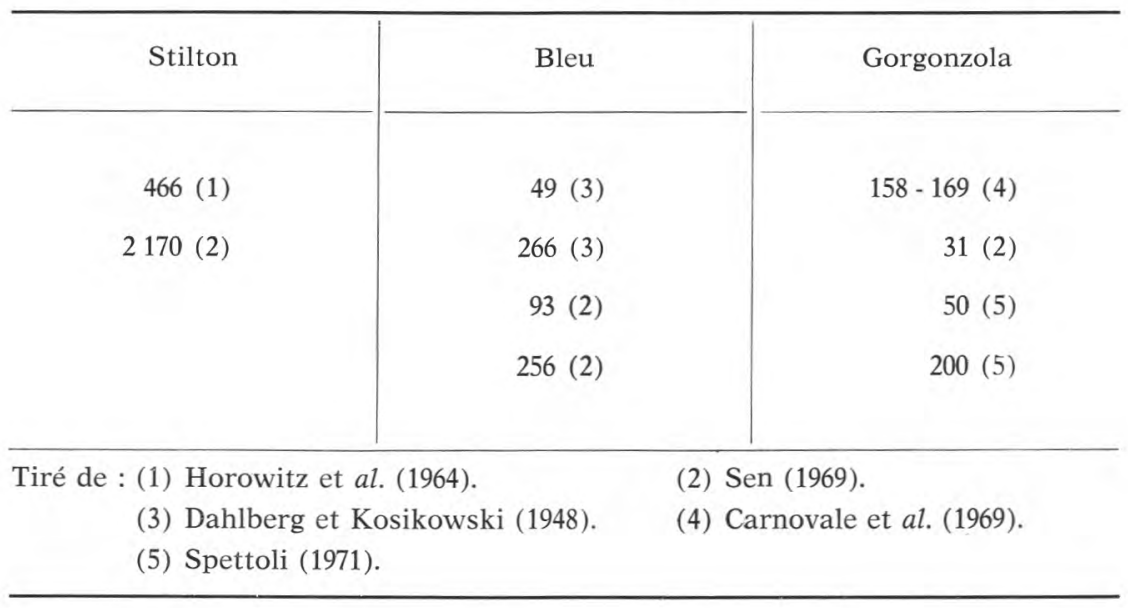


En ce qui concerne l'histamine, Spettoli (1971) publie deux valeurs obtenues par fluorimétrie sur 2 échantillons de Gorgonzola (50 et $450 \mu \mathrm{g} / \mathrm{g}$ ).

\section{CONCLUSION}

En conclusion, si l'on se place du point de vue de l'état actuel des connaissances, on peut opposer les pâtes molles sensu stricto aux pâtes persillées.

Il apparaît que les premières, en dépit de leur importance économique considérable, ont été passablement délaissées. Dans ce domaine, les connaissances sont des plus restreintes notamment au niveau des composés neutres et basiques. De plus, la rareté des données quantitatives rend hasardeuse la recherche de corrélations avec les observations organoleptiques. Assurément, il y a là un domaine presque inexploré qui se prête à de nombreuses recherches originales.

Pour les pâtes persillées, au contraire, les données chiffrées sont abondantes. Il faut probablement voir là une conséquence de l'utilisation de ces fromages en tant que condiment aromatique. Dans ce secteur, le stade de la recherche purement descriptive a été dépassé puisque l'on a pu voir apparaître des produits d'imitation sur le marché (Nelson, 1970).

\section{S u m m a r y}

In cheese of the washed rind and of the mouldy type rind considerable lipolysis occurs but there is a selective release of long chain fatty acids. As to volatile fatty acids, they have been clearly identified in a large number of cheese varieties. Levels of each of such acids vary considerably from one variety to the next and rather significant variations are found between samples of the same variety of similar organoleptic quality. Therefore, it appears that these acids do not decisively determine the flavour.

Very little work has been done on neutral compounds (carbonyls, alcohols, esters). The sulphur compounds appear to be particularly important in washed rind cheese. Data on amino acids are relatively abundant but it does not seem that they are determinant in flavour development. Information on volatile amines are practically non existent.

In blue cheese acids are recognized to play an important role together with methyketones. When available in relatively large amounts these ketones are reduced to seconday alcohol whose organoleptic function is not well defined. Others compounds among 
which amino acids and amines have been identified and sometimes determined but their role at the concentration available is not well known.

Reçu pour publication le 15 octobre 1973.

\section{Bibliographie}

Anderson (D. F.) (1966). - Flavor chemistry of blue cheese. Diss. Abstr, 26, (6), 6636-37.

ANDERson (D. F.) and DAY (E. A.) (1965). - Quantitative analysis of the major free fatty acids in blue cheese. J. Dairy Sci., 48, (2), 248-49.

Benassi (R.) (1964), - Lipolysis in cheese. Latte, 38, (4), 335-38.

BULINSKI (R.) (1969). - Untersuchungen über den Gehalt an biologisch aktiven Aminen in Lebensmittelnzesz. Milchwiss., 24, 697.

Carnovale (E.), Fratoni (A.), Lintas (C.) (1969). - Studi sulla determinazione della tiramina in diverse varieta di formaggi. Boll. S.I.B.S., 45, 667, cité par Cerruti (G.), Zappavigna (G.) (1970). Il Latte, 44, (10), 707-714.

Coffman (S. R.), Smith (D. E.) and Andrews (J. S.) (1960). - Analysis of volatile food flavors by G.L.C.I. The volatile compounds from dry blue cheese and dry Romana cheese. Food Res., 25, 663.

Csiszar (Jr.), Tomka (G.) und Romlehnes-Bakos (Anna) (1956). - Die bildung von Aromastoffen (Acetoin + Diacetyl) im Käse und Einfluss auf die Qualität der Käse. 14c Int. Dairy Congr., 2, (2), 125-45.

Dahlberg (A. C.) and Kosikowski (F. V.) (1948). - The tyramine of cheese. J. Dairy Sci., 31, 293.

DaRTEy (C. K.) and Kinsella (J. E.) (1971), - Rate of formation of methyl ketones during blue cheese ripening. J. Agric. Fd Chem., 19, (4), 771-74.

DAY (E. A.) and ANDERson (D. F.) (1965), - Gas chromatographic and mass spectral identification of natural components of the aroma fraction of blue cheese. J. Agric. Fd Chem., 13, (1), 2-4.

DolevaleK (J.) and Brabcova' (Jarmila) (1964). - Methyl ketones in mould-ripened cheese during ripening. Sb. vys. Sk. chem. technol. Praze, Potravin. Technol., 8, (2), 197-208. D.S.A., 28, 3058 (1966).

Do Ngoc (M.), Lenoir (J.) et Choisy (C.) (1971). — Les acides aminés libres des fromages affinés de Camembert, Saint-Paulin et Gruyère de Comté. Rev. Lait. Franç., juin 1971, 288, 447-62.

Dumont (J. P.) et AdDA (J.). - Résultats non publiés.

Grill (H.) Jr., PAtTon (S.) and CONE (J. F.) (1966). - Aroma significance of sulfur compounds in surface ripened cheese. J. Dairy Sci., 49, (4), 409-13.

Grill (H.), Patton (S.) and Cone (J. F.) (1966). - Methyl mercaptan and hydrogen sulfide as important flavour components of Trappist-type cheese. J. Dairy Sci., 49, (6), 710.

Hanni ( $\mathrm{H}_{3}$ ) (1967). - Biochemical processes in cheese. Schw. Milch., 93, (5-8), $442-44$.

Hiscox (E. R.), Harrison (J.) and Wolf (J.Z.) (1951). - The ripening of Stilton cheese : changes in the volatile acid content. J. Dairy Res., 18, 296.

Horowitz (D.), Lovenberg (W.), Engelman (K.), Suoerdsna (A.) (1964). - Monoamine oxidase inhibitors, tyramine and cheese. J.A.M.A., 188, 1108. 
Hote-Baudart (E.) (1967). - Acides gras libres du Camembert. Bull. Rech. agron. Gembloux II, 4, 667-683.

JACKSON (H. W.) and Hussong (R. V.) (1958). - Secondary alcohols in blue cheese and their relation to methyl ketones. J. Dairy Sci., 41, 920.

JACQUet (S.) et LenOIR (S.) (1954). - Recherches sur la libération des acides aminés au cours de l'affinage des Camemberts de Normandie. C.R. Acad. Sct Paris, $238,(23), 2265-66$.

KIERMEIR (F.) und JARCZYNSKI (R.) (1960). - Uber die quantitative Bestimmung von p-Hydroxybenzoesäure in Käse. Z. Lebensm. Untersuch. u. Forsch, 113, 370.

Kosikowski (F. V.) and DaHLberg (A. C.) (1954). - A quantitative appaisal of the free amino acids in foreign type cheese. J. Dairy Sci., 37, 167.

Kuzdzal-Savoie (S.) et Kuzdzal (W.) (1966). - Contribution à l'étude de la lipolyse dans les fromages. La Technique Laitière, 14, 17-20.

Kuzdzal (W.) et KuzDzal-Savoie (S.) (1966). - Etude comparée des acides gras non-volatils libres et estérifiés dans les fromages. $17^{\mathrm{e}}$ Congr. Int. Lait., D.2., 335-342.

KuzDzaL-SavoIE (S.) et AdDa (J.) (1970). - L'arôme de quelques types de fromages français. La France et ses parfums, $\mathrm{n}^{\circ} 69$.

KuzdzaL-Savoie (S.) et Kuzdzal. (W.) (1971). — Dosage des acides gras libres volatils dans quelques types de fromages français. La Technique Laitière, $\mathrm{n}^{\circ} 717$.

KuzdzaL-Savoie (S.), Losi (G.), Kuzdzal (W.) et Goto (K.) (1971). - Mise en évidence des acides benzoïque, phénylacétique et phénylpropionique dans les acides gras libres du Munster et du Livarot. La Technique Laitière, $\mathrm{n}^{\circ} 724$.

Kuzdzal-Savoie (S.), Kuzdzal (W.), Trehin (J.) et Goto (K.) (1971). - Les acides gras libres du Munster. Rev. Lait. Franç., $\mathrm{n}^{\circ}$ 290, 615-29.

LeNoIr (J.) (1963). - Note sur la composition en matières azotées des fromages affinés de Camembert, Saint-Paulin et Gruyère de Comté. Le Lait, 43, (428), 500-07.

Masson (J. D.) et Panouse (J. J.) (1968), - Bull. Soc. Chim. Franç., 7B, 48.

Nelson (J. H.) (1970). - Blue cheese flavor by fermentation. Food product development. Feb.-March.

NeY (K. H.) und Wirotama (I. P. G.) (1971). - Aliphatic monoamine in deutschem und französischem Camembert. Z. Lebensmit. Untersuch. Forsch., 146, 343-44.

Ney (K. H.) und Wiromata (I. P. G.) (1972). - Untersuchung von EdelpilzkäseAroma. Z. Lebensmitt. Untersuch., 149, 175-279.

Niki (T.), Yoshioka (Y.) and Aнiкo (K.) (1966). - Proteolytic and lipolytic activities of Penicillium roqueforti isolated from blue cheese. XVII Int. Dairy Congr. D., 531-37.

Patton (S.) (1950). - The methyl ketones in blue cheese and their relation to its flavor. J. Dairy Sci., 33, 680.

Ramshaw (E.) et ADDA (J.). - Résultats non publiés.

Sato (M.), Honda (T.), Yamada (Y.), Takada (A.) and Kawanami (T.) (1966). - A study on free fatty acids, volatile carbonyl compounds and tyrosine in blue cheese. XVII Int. Dairy Congr. D., 539-44.

SCHörmuller (J.) und LANGNER (M.) (1960). - Uber die organischen Säuren verschiederer Käsearten. Z. Lebensm. Untersuch. u. Forsch, 113, 289.

Schwartz (D. P.) and PARKS (O. W.) (1963). - Quantitative analysis of methyl ketones in blue cheese fat. J. Dairy Sci., 46, (9), 989-90.

Schwartz (D. P.) and Parks (O. W.) (1963). - Methyl ketones in Camembert cheese. J. Dairy Sci., 46, (10), 1136. 
SEN (N. P.) (1969). - Analysis and significance of tyramine in foods. J. Food Sc., $34,22$.

Simonart (P.) et Mayaudon (J.) (1952). - Etude chromatographique de fromages. I. Analyse qualitative des substances ninhydrine positives. Neth Milk and Dairy J., 6, 1.

SimonaRt (P.) et MaYAudon (J.) (1956). - Formation d'acides aromatiques et aliphatiques au cours de la maturation des fromages du type "Limbourg ". 14 th. Intern. Dairy Congr., Rome, 2, 541.

SimonaRt (P.) et Mayaudon (J.) (1956). - Etude chromatographique des fromages. II. Acides alphatiques. III. Acides aromatiques. Neth. Milk Dairy J., 10, $156-261$.

Spettoli (P.) (1971). - Contenuta in amine biogene di 24 formaggi italiani. Ind. $A g r_{\circ}, I X,(2), 42-46$.

Stark (W.) et AdDa (J.) (1972). - Acides gras volatils du Livarot.et du Pont-l'Evêque. La Technique Laitière, $\mathrm{n}^{\circ} 746$.

Svensen (A.) and OtTeSTAD (E.) (1969). - Qualitative and quantitative analysis of flavour components in Normanna cheese. Meieriposten, 58, (3), 50-57, (4), 77-81.

TuckeY (S. L.) and SAHASRABudHe (M. R.) (1957). - Studies in the ripening of Limburger cheese. J. Dairy Sci., 40, 1329.

Tuckey (S. L.), Colmey (J.) et Azuma (Y.) (1959). - Formation of carbonyls during ripening of Limburger cheese. J. Dairy Sci., 42, (5), 901-02 (Abstr.).

Tsugo (T.), Hayashi (T.) and Matsuoka (H.) (1959). - Studies on the manufacture of a semi soft cheese ripened by Penicillium casei colum. XVth. Int. Dairy Congr., 2, (3), 899. 